\title{
Circulation Journal Awards for the Year 2010
}

\author{
Hiroaki Shimokawa, MD, PhD
}

Dear Colleagues:

On behalf of the Editorial Team, I am pleased to announce the Circulation Journal Awards for the Year 2010 .

The aim of these Awards is to recognize papers published in 2010, both clinical and experimental studies, that were highly appreciated by the Editorial Team. The selection process comprises 2 steps. First, from the 277 original papers published in the Journal in 2010, our 33 Japanese Associate Editors selected papers with a high scientific level in their respective fields, and second, the 2 Associate Editorial Teams (16 on 1 team and 17 on the other) further evaluated the selected papers in terms of originality, contribution to cardiovascular science, manner of paper preparation, and future possibilities.

In the year of 2010, the following 5 papers have been selected for the Circulation Journal Awards.

\section{$<$ First Place in the Clinical Investigation Section >}

\section{Complete Elimination of Cardiodepressant IgG3 Autoantibodies by Immunoadsorption in Patients With Severe Heart Failure \\ Akiyasu Baba, Makoto Akaishi, Megumi Shimada, Toshiaki Monkawa, Yasuhisa Wakabayashi, Michiko Takahashi, Yuji Nagatomo, Tsutomu Yoshikawa. \\ (Departments of Cardiology and Nephrology, Kitasato Institute Hospital, Kitasato Uni- versity School of Medicine, Tokyo and Departments of Nephrology and Cardiology, Keio University School of Medicine, Tokyo, Japan)}

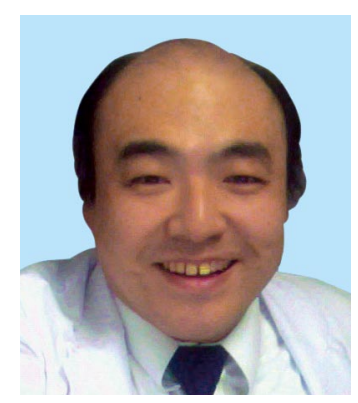

Background: Cardiodepressant IgG3 autoantibodies (CD-Abs) can be targeted by apheresis. Using blinded measurements of CD-Abs before and after immunoadsorption (IA), the cardiac function of patients who did or did not achieve complete CD-Abs elimination was compared.

Methods and Results: Autoantibodies were completely removed from 18 patients with heart failure (New York Heart Association class 3 or 4, left ventricular ejection fraction (LVEF) <30\%) using a selective IgG3 adsorption column. All patients had anti- $\beta 1$-adrenergic and/or M2-muscarinic autoantibodies before IA, and all LVEF were measured on radionuclide ventriculography. CD-Abs were measured before and after IA, and patient status was blinded until all measurements were collected. Treatment was defined as complete when $\mathrm{CD}$-Abs status changed from positive to negative after IA. Other instances were defined as incomplete. Six-min walk test results and brain natriuretic peptide levels improved significantly after IA $(\mathrm{P}<0.01)$. The increase in LVEF 3 months after IA was significantly greater after complete treatment in comparison to the incomplete treatment group $(19 \pm 8-29 \pm 9 \%$ vs. $18 \pm 9-17 \pm 8 \%, \mathrm{P}<0.01)$. Cardiac insufficiency events were also more frequent in the incomplete treatment group.

Conclusions: Complete elimination of CD-Abs with apheresis may be related to improved cardiac function in the treatment of heart failure. ${ }^{1} \quad$ (Circ J 2010; 74: 1372-1378)

[Comment] The number of patients with heart failure has been rapidly increasing worldwide. Although significant progress has been made in heart failure practice, more effective therapies remain to be developed. Elimination of cardiodepressant IgG3 autoantibodies (CD-Abs) is one of the promising new therapies for heart failure. In this study, the authors were able to demonstrate for the first time that there is a close correlation between the extent of CD-Abs elimination and that of improvement of cardiac function, suggesting the usefulness of the new therapy. 
$<$ Second Place in the Clinical Investigation Section >

\section{Trends in Acute Myocardial Infarction Incidence and Mortality Over 30 Years in Japan - Report From the MIYAGI-AMI Registry Study - \\ Toru Takii, Satoshi Yasuda, Jun Takahashi, Kenta Ito, Nobuyuki Shiba, Kunio Shirato, Hiroaki Shimokawa on behalf of the MIYAGI-AMI Study Investigators. (Department of Cardiovascular Medicine, Tohoku University Graduate School of Medicine, Sendai, Japan)}

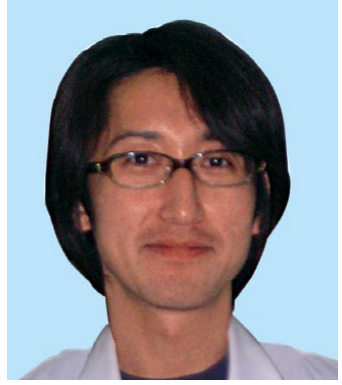

Background: Worldwide, the rate of aging is highest in Japan, especially the female population. To explore the trends for acute myocardial infarction (AMI) in Japan, the MIYAGI-AMI Registry Study has been conducted for 30 years since 1979, whereby all AMI patients in the Miyagi prefecture are prospectively registered.

Methods and Results: In 1979-2008, 22,551 AMI patients (male/female 16,238/6,313) were registered from 43 hospitals. The age-adjusted incidence of AMI (/100,000 persons/year) increased from 7.4 in 1979 to 27.0 in $2008(\mathrm{P}<0.001)$. Although control of coronary risk factors remained insufficient, the rates of ambulance use and primary percutaneous coronary intervention (PCI) have increased, and the overall in-hospital mortality (ageadjusted) has decreased from $20.0 \%$ in 1979 to $7.8 \%$ in 2008 ( $\mathrm{P}<0.0001)$. However, the in-hospital mortality remains relatively higher in female than in male patients ( $12.2 \%$ vs. $6.3 \%$ in 2008). Female patients were characterized by higher age and lower PCI rate.

Conclusions: The MIYAGI-AMI Registry Study demonstrates the steady trend of an increasing incidence, but decreasing mortality, for AMI in Japan over the past 30 years, although the female population still remains at higher risk for in-hospital death, despite improvements in the use of ambulances and primary PCI. ${ }^{2}$ (Circ $J$ 2010; 74: $93-100$ )

[Comment] The MIYAGI-AMI Registry Study is the only registry study in Japan where all AMI patients have been prospectively registered in a prefecture level for more than 30 years. In this study, the investigators analyzed the 30-year data and found that the incidence of AMI has been steadily increasing but that of in-hospital death has been decreasing, to which improvements in the use of ambulances and primary PCI may be contributed. This study could explain in part why the number of patients with chronic heart failure has been rapidly increasing in Japan.

\section{$<$ Second Place in the Clinical Investigation Section $>$}

\section{Tissue Characterization of Coronary Plaques and Assessment of Thickness of Fibrous Cap Using Integrated Backscatter Intra- vascular Ultrasound - Comparison With Histology and Optical Coherence Tomography - \\ Masanori Kawasaki, Arihiro Hattori, Yoshiyuki Ishihara, Munenori Okubo, Kazuhiko Nishigaki, Genzou Takemura, Masanao Saio, Tsuyoshi Takami, Shinya Minatoguchi. \\ (Regeneration \& Advanced Medical Science and Department of Immunopathology, Gifu University Graduate School of Medicine, Gifu, Japan)}

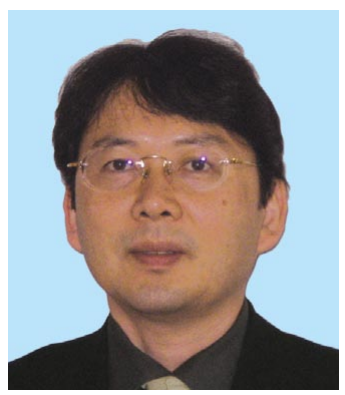

Background: The purpose of this study was to develop a new online integrated backscatter intravascular ultrasound (IB-IVUS) system and to validate its ability to measure fibrous cap thickness by comparing IB-IVUS images with those from optical coherence tomography (OCT).

Methods and Results: Images were acquired from 125 segments of 26 coronary arteries obtained at autopsy from 11 cadavers. In the training study $(n=30), 242$ regions-of-interest on color-coded maps were compared with histology. In the validation study, 95 cross-sections were diagnosed by IB-IVUS and histology. In 24 patients with stable angina, 28 arterial cross-sections were imaged by IB-IVUS and OCT in vivo. In the training study, cutoff values of -39 decibels $(\mathrm{dB})$ and $-17 \mathrm{~dB}$ were the optimal predictors of lipid pool/fibrosis and fibrosis/calcification, respectively, with $38-\mathrm{MHz}$ mode; $-42 \mathrm{~dB}$ and $-20 \mathrm{~dB}$, respectively, with $43-\mathrm{MHz}$ mode. In the validation study, IB classified the fibrous, lipid-rich and fibrocalcific components with an accuracy of 92\%, 91\% and 95\%, respectively. Agreement between the histological and IB-IVUS diagnoses was excellent 
(Cohen's $\kappa=0.83$ ). There was a correlation between the fibrous cap thickness measured by IB-IVUS and OCT $(\mathrm{r}=0.74, \mathrm{P}<0.001)$.

Conclusions: The IB-IVUS system with improved resolution provides high diagnostic accuracy for the analysis of the tissue characteristics of coronary plaques, and enables estimation of the thickness of the fibrous cap in the clinical setting. ${ }^{3}$ (Circ J 2010; 74: 2641-2648)

[Comment] Recently, significant progress has been made in cardiovascular imaging, including the development of the integrated backscatter intravascular ultrasound (IB-IVUS) system. In this study, the authors developed a new online IB-IVUS system and were able to validate its high diagnostic accuracy for tissue characterization of coronary plaques and assessment of the thickness of the fibrous cap by comparing IB-IVUS images with those from optical coherence tomography.

\section{$<$ First Place in the Experimental Investigation Section $>$}

\section{Tongue Muscle-Derived Stem Cells Express Connexin 43 and Improve Cardiac Remodeling and Survival After Myocardial Infarction in Mice \\ Masaki Shibuya, Toshiro Miura, Yasuhiro Fukagawa, Shintaro Akashi, Takamasa Oda, Shuji Kawamura, Yasuhiro Ikeda, Masunori Matsuzaki. \\ (Division of Cardiology, Department of Medicine and Clinical Science, Yamaguchi Univer- sity Graduate School of Medicine, Ube, Japan)}

Background: Cell transplantation therapy for heart failure is hindered by poor differentiation into cardiomyocytes and arrhythmias caused by the poor expression of connexin 43 (Cx43). A new stem cell source for cardiac regeneration is needed.

Methods and Results: Tongue muscle-derived Sca-1(+) cells (TDSCs) were isolated from normal and green fluorescence protein (GFP)-transgenic mouse tongues using surface antigen Sca-1. Cardiomyogenic differentiation was confirmed by measuring the calcium transient and the expression of cardiac-specific genes. The formation of gap junctions was confirmed by the expression of $\mathrm{Cx} 43$ and the dye transfer method. The contraction of regenerated cells was demonstrated by the calcium transients. GFP mouse-derived TDSCs were transplanted into hearts in a model of acute myocardial infarction. Three months after transplantation, LV remodeling was attenuated and the survival rate was improved compared with the control group.

Conclusions: TDSCs form gap junctions and improve cardiac function and long-term survival after myocardial infarction. ${ }^{4} \quad$ (Circ J 2010; 74: 1219-1226)

[Comment] Cell therapy for heart failure has attracted much attention; however, the source of potential cardiomyocytes is limited partly due to poor expression of connexin 43 (Cx43). This study is unique because the authors were able to demonstrate that transplantation of tongue muscle-derived Sca-1(+) cells, which express $\mathrm{Cx} 43$ and thus form myocardial gap junctions, was useful for ameliorating survival and cardiac function in a mouse model of acute myocardial infarction in vivo.

\section{<Second Place in the Experimental Investigation Section>}

\section{Evidence for the Therapeutic Potential of Ex Vivo Expanded Human Endothelial Progenitor Cells Using Autologous Serum}

Takanori Shumiya, Rei Shibata, Yuuki Shimizu, Masakazu Ishii, Ryuji Kubota, Satoshi Shintani, Toyoaki Murohara.

(Department of Cardiology, Nagoya University Graduate School of Medicine, Nagoya, Japan)

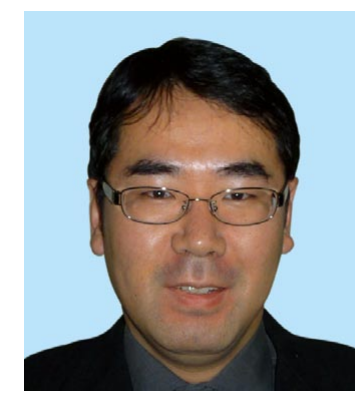

Background: Transplantation of endothelial progenitor cell (EPC) augments angiogenesis in animal models of tissue ischemia. Although it is desirable to use expanded autologous EPCs for therapeutic angiogenesis in the 
clinical arena, a major obstacle is the limitation of the EPC expansion technique without using animal-derived serum such as fetal bovine serum (FBS). To overcome this issue, the possibility of human EPC (hEPCs) expansion using autologous serum (AS) culture was investigated.

Methods and Results: Peripheral blood mononuclear cells were isolated from healthy volunteers by densitygradient centrifugation and culture-expanded in medium containing either FBS or AS. In vitro angiogenic functions, such as differentiation, migration and tube formation, were not significantly different between hEPCs cultured with FBS and AS. Next, we investigated whether transplantation of hEPCs would augment angiogenesis in unilateral hind limb ischemia using nude mice. The ratio of ischemic/normal limb blood flow and tissue capillary density in mice receiving hEPCs cultured with either FBS or AS significantly increased as compared with control mice receiving PBS alone. The ischemic/normal limb blood flow ratio and histological capillary density were not significantly different between hEPCs cultured with FBS and AS.

Conclusions: hEPCs can be culture-expanded in medium containing AS. Moreover, the angiogenic functions of such hEPCs are almost identical to those of hEPCs cultured with FBS. Ex vivo expanded hEPCs using AS seems to be useful for future clinical therapeutic angiogenesis. ${ }^{5} \quad$ (Circ J 2010; 74: 1006-1013)

[Comment] Although the potential usefulness of endothelial progenitor cells (EPC) has been repeatedly demonstrated, one of the limitations of the therapy is the use of animal-derived serum for cell culture. In this study, the authors were able to demonstrate that human EPC (hEPC) can be cultured and expanded with autologous serum with the same potency and effectiveness in vivo as compared with fetal bovine serum. The present method could further facilitate the cell therapy with hEPC for various ischemic disorders.

Awards will be presented to the 5 research groups during the 75 th Annual Scientific Meeting of the Japanese Circulation Society, and will also be announced on the Society website. We look forward to receiving manuscripts with high scientific impact for publication in the Circulation Journal in 2011.

Hiroaki Shimokawa, MD, PhD

Editor-in-Chief

Circulation Journal

(Released online February 11, 2011)

\section{References:}

1. Baba A, Akaishi M, Shimada M, Monkawa T, Wakabayashi Y, Takahashi M, et al. Complete elimination of cardiodepressant IgG3 autoantibodies by immunoadsorption in patients with severe heart failure. Circ $J$ 2010; 74: 1372-1378.

2. Takii T, Yasuda S, Takahashi J, Ito K, Shiba N, Shirato K, et al; MIYAGI-AMI Study Investigators. Trends in acute myocardial infarction incidence and mortality over 30 years in Japan: Report from the MIYAGI-AMI Registry Study. Circ J 2010; 74: 93 100.

3. Kawasaki M, Hattori A, Ishihara Y, Okubo M, Nishigaki K, Takemura G, et al. Tissue characterization of coronary plaques and assessment of thickness of fibrous cap using integrated backscatter intravascular ultrasound: Comparison with histology and optical coherence tomography. Circ J 2010; 74: 2641-2648.

4. Shibuya M, Miura T, Fukagawa Y, Akashi S, Oda T, Kawamura S, et al. Tongue muscle-derived stem cells express connexin 43 and improve cardiac remodeling and survival after myocardial infarction in mice. Circ J 2010; 74: 1219-1226.

5. Shumiya T, Shibata R, Shimizu Y, Ishii M, Kubota R, Shintani S, et al. Evidence for the therapeutic potential of ex vivo expanded human endothelial progenitor cells using autologous serum. Circ J 2010; 74: 1006-1013. 